\title{
Modelling the Electromagnetic Behaviour of SiFe Alloys Using the PreisachTheory and the Principle of Loss Seperation
}

\author{
L. DUPRÉ ${ }^{a, *}$, R. VAN KEER $^{b}$ and J. MELKEBEEK ${ }^{a}$ \\ ${ }^{\text {a Department }}$ of Electrical Engineering, \\ ${ }^{b}$ Department of Mathematical Analysis, Ghent University, \\ B-9000 Gent, Belgium
}

(Received 9 August 2000)

In this paper we present 2 simplified methods for the evaluation of magnetisation loops in laminated SiFe alloys, using the Preisach theory and the statistical loss theory. These methods are investigated in detail as a practical alternative for a very accurate, but much involved numerical approach, viz. a combined lamination model - dynamic Preisach model earlier developed by the authors. Particularly, one of the 2 methods provides accurate results inspite of a dramatic reduction of the CPU-time in comparison with the earlier developed combined model. For the other simplified method, the reduction of CPU-time is less pronounced but still considerable and the results are fairly good.

Keywords: Magnetic materials; Hysteresis; Dynamic Preisach model; Statistical loss theory; Static and dynamic field

AMS Classifications: 35K55, 65M05, 65N30, 72A25

\section{INTRODUCTION}

The Preisach theory [1] combined with Maxwell equations [2] has received considerable attention over many years, because it includes

\footnotetext{
* Corresponding author. Department of Electrical Power Engineering, Laboratory for electrical machines and power electronics, St. Pietersnieuwstraat 41, B-9000 Gent, Belgium. Tel.: + 32926434 24, Fax: + 32926435 82, e-mail: luc.dupre(a),rug.ac.be
} 
many key features of the quasi-static as well as of the dynamic behaviour of magnetic alloys in a mathematically elegant way. In [3], a rate-dependent Preisach model, denoted by DPM, was introduced by assuming that the switching of each elementary Preisach dipole cannot occur instantaneously, but at a finite rate controlled by the difference between the external field and the loop threshold fields. In [3], it was shown that the area of the loop predicted by the DPM follows a law of the form $C_{0}+C_{1} \sqrt{f}$ in terms of the imposed frequency $f$. In this paper, we present and evaluate two simplified models as an alternative for the complex combined lamination-dynamic Preisach model, described in detail in [4-6]. These simplified models have the advantage to predict the material behaviour in a more efficient way with respect to CPUtime, however with a relatively small loss of accuracy.

\section{STATISTICAL LOSS THEORY}

A general approach to the calculation of electromagnetic losses in soft magnetic laminated materials under unidirectional flux $\varphi(t)$ is based on the separation of the losses into three components: the hysteresis losses $P_{h}$, the classical losses $P_{c}$ and the excess losses $P_{e}$. According to the statistical loss theory [7], the magnetisation process in a given cross section $S$ of the magnetic lamination of thickness $d$ can be described in terms of $n$ simultaneously active correlation regions. For several alloys, $n$ is a linear function of the excess field $H_{\mathrm{exc}}=P_{e} /\left(4 f B_{m}\right)$, i.e.,

$$
n=H_{\text {exc }} / V_{0}
$$

When (1) holds, the total losses under a sinusoidal flux excitation with frequency $f$ and maximum induction $B_{m}$, can be written as, $c f$. [7]

$$
\begin{aligned}
P_{t}=P_{h}+P_{c}+P_{e} \equiv & W_{h}\left(B_{m}\right) f+\frac{1}{6} \sigma \pi^{2} d^{2} B_{m}^{2} f^{2} \\
& +8 \sqrt{\sigma G S V_{0}\left(B_{m}\right)}\left(B_{m} f\right)^{(3 / 2)}
\end{aligned}
$$

Here, $\sigma$ is the electrical conductivity and $G=0.1357$. Notice the specific frequency dependency for $P_{h}, P_{c}$ and $P_{e}$. The fitting parameters $W_{h}$ and $V_{0}$, depending on $B_{m}$, are defined by the microstructure of the material. 


\section{THE DYNAMIC PREISACH THEORY}

The scalar classical Preisach model (CPM) [1] provides quite an accurate description of hysteresis effects in magnetic materials. In this model, each Preisach dipole has a rectangular non symmetric hysteresis loop defined by two characteristic parameters $\alpha$ and $\beta(\beta \leq \alpha)$. Being dependent on the history of the magnetic field, the magnetisation of the dipole, denoted by $\phi$, takes the value +1 or -1 , according to

$$
\phi=\left\{\begin{array}{l}
+1: H_{e f}(t)>\alpha \text { or }\left(\beta<H_{e f}<\alpha \text { and } H_{\text {last }}>\alpha\right) \\
-1: H_{e f}(t)<\beta \text { or }\left(\beta<H_{e f}<\alpha \text { and } H_{\text {last }}<\beta\right) .
\end{array}\right.
$$

Here $H_{\text {last }}$ is the last extreme value kept in memory outside the interval $[\beta, \alpha]$.

In the dynamic Preisach model (DPM) [3], the switching rate of each dipole is given by:

$$
\frac{d \phi}{d t}= \begin{cases}k_{d}\left(H_{e f}(t)-\alpha\right) & H_{e f}(t)>\alpha \text { and } \phi<+1 \\ k_{d}\left(H_{e f}(t)-\beta\right) & H_{e f}(t)<\beta \text { and } \phi>-1 \\ 0 & \text { in the other cases }\end{cases}
$$

where $k_{d}$ is a material parameter. Then, in the case of CPM as well as DPM, the induction $B(t)$ is obtained by an integral in the $(\alpha, \beta)$-plane:

$$
B(t)=B_{\text {rev }}\left(H_{e f}(t)\right)+\frac{1}{2} \int_{-\infty}^{\infty} d \alpha \int_{-\infty}^{\alpha} d \beta \phi(\alpha, \beta, t) P(\alpha, \beta) .
$$

Here, $P(\alpha, \beta)$ is the Preisach distribution function, describing the hysteresis behavior, and the effective field $H_{e f}(t)$ is obtained from the applied field $H(t)$ and the corresponding magnetisation $B(t)$, viz. $H_{e f}(t)=H(t)+k_{m} B(t)$. In the CPM, the $(\alpha, \beta)$-plane is divided into two regions in which $\phi$ equals +1 and -1 depending on the history of $H_{e f}$. In the DPM, the $(\alpha, \beta)$-plane is divided into 3 subregions, i.e., two regions in which $\phi$ equals +1 and -1 respectively, and a third region, in which the dipoles are in an intermediate state $(-1<\phi<+1)$. The shape of the third region is defined by the time variation of the magnetic field. For quasi-static flux excitations $(f \rightarrow 0)$, this region reduces to the same polygonal as in the CPM-case, seperating the subregions 1 and 2 . 
It is shown in [3] that the finite rate $k_{d}$ of switching of the dipoles results in an increase of the area enclosed by the BH-loops. Moreover, the dynamic losses obtained by this DPM are found to vary with $f^{(3 / 2)}$, as in the third term of the RHS of (2), under a sinusoidal flux excitation and thus may describe the hysteresis and excess losses.

\section{THE COMBINED LAMINATION-DYNAMIC PREISACH MODEL}

In order to be able to describe correctly the three types of losses occuring in magnetic alloys and their interplay due to the interaction between the induced eddy currents and the magnetic material characteristics, the Preisach theory must be combined with macroscopic field calculations in the laminated magnetic material. Throughout the lamination, shown in Figure 1, which is assumed to be isotropic, the time dependent flux $\varphi$ flows in the $z$-direction and the magnetic field has only one component, viz. $\bar{H}=H \cdot \bar{e}_{z}$. As $d \ll w$, by eliminating the edge effects, we may assume $H$ to vary in the

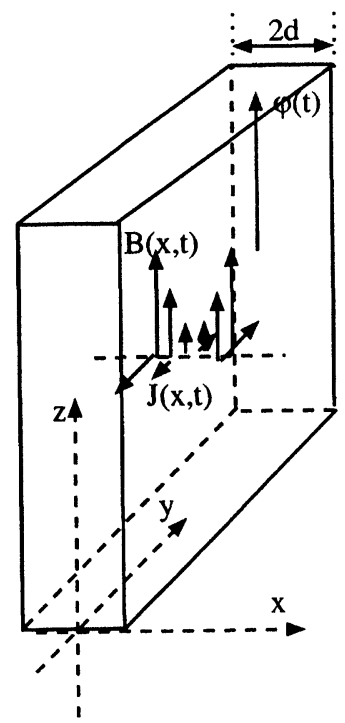

FIGURE 1 The combined lamination model - dynamic Preisach model. 
$x$-direction only. From the Maxwell equations the governing equation for $H$ reads:

$$
\frac{1}{\sigma} \frac{\partial^{2} H}{\partial x^{2}}=\frac{\partial B}{\partial t}
$$

where $B$ is the magnitude of the magnetic induction $\bar{B}=B(x) \cdot \bar{e}_{z}$, while $\sigma$ is the electrical conductivity of the material. Due to this conductivity, eddy currents $\bar{J}$ are generated in the material. By these eddy currents the flux is no longer distributed uniformly over the lamination, but is concentrated at the edges of the lamination. This is called skin effects, [8]. For each value of $x$, the relation between $B$ and $H$ is given either by (3),(5) or by (4),(5).

To obtain a well posed boundary value problem (BVP), (6) must be completed with appropriate boundary conditions (BCs) and initial conditions (ICs), viz.

$$
\frac{\partial H(x=0, t)}{\partial x}=0, \frac{\partial H(x=d, t)}{\partial x}=\frac{\sigma}{2} \frac{d \varphi}{d t}
$$

and

$$
H(x, t=0)=0,\left\{\begin{array}{l}
\phi(\alpha, \beta, t=0)=+1: \alpha+\beta<0 \\
\phi(\alpha, \beta, t=0)=-1: \alpha+\beta>0
\end{array}, \quad \forall x \in[0, d]\right.
$$

The first $\mathrm{BC}$ reflects the symmetry in the lamination. The second $\mathrm{BC}$ follows when combining (6) with the symmetry and with the definition of the flux $\varphi(t)$ through the lamination. Finally, the IC (8) corresponds to the demagnetized state of the material. For the numerical solution of the complete BVP (4)-(8), we refer to [5]. The combined lamination model-dynamic Preisach model (LM-DPM) consists of the BVP (6)(8) in which the relation between the induction $B$ and the field strength $\mathrm{H}$ is described by means of (4)-(5).

The total losses in the magnetic material is then obtained by the area enclosed by the $B_{a} H_{s}$-loop, where $B_{a}$ and $H_{s}$ are the induction averaged out over the cross section of the lamination and the magnetic field at the surface of the lamination respectively, i.e.,

$$
B_{a}(t)=\frac{1}{d} \int_{0}^{d} B(x, t) d x=\frac{\varphi(t)}{2 d}, H_{s}(t)=H(d, t)
$$




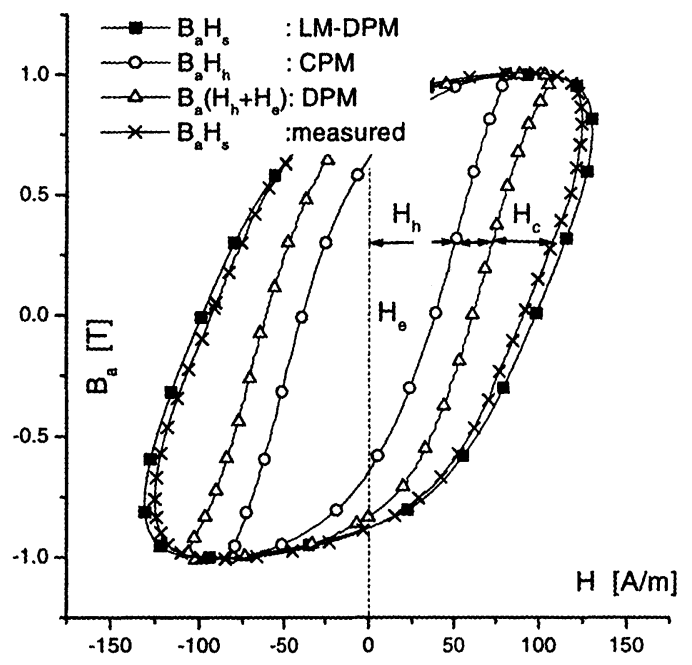

FIGURE 2 Comparison between the magnetisation loops from the classical Preisach model, from the dynamic Preisach model, from the LM-DPM and measurements; definition of the fields $H_{h}, H_{e}$ and $H_{c}$.

Figure 2 compares the numerically obtained $\mathrm{BH}$-loops, when using the CPM (quasi-static loop defined by $H_{h}$ : open circle line), when using the DPM (dynamic loop defined by $H_{h}+H_{e}$ : open triangle line), and when using the LM-DPM (dynamic loop defined by $H_{h}+H_{e}+H_{c} \equiv H_{h}+$ $H_{d}$ : solid square line) with the measured BH-loop (crosses-line) in the case of a sinusoidal flux of $100 \mathrm{~Hz}$ and 1 Tesla. Notice the good correspondence between the loop obtained by the LM-DPM and the measured BH-loop. The discrepancy between the open circle line and the measured $\mathrm{BH}-\mathrm{loop}$ results from the neglection of the excess and the classical losses in the CPM, while the discrepancy between the open triangle line and the measured $\mathrm{BH}$-loop is due to neglecting the classical losses.

\section{SIMPLIFIED MODELS}

From (2), it is observed that, under sinusoidal flux excitation, the classical losses and the excess losses are depending on $\left(B_{m} f\right)^{2}$ and $\left(B_{m} f\right)^{(3 / 2)}$ respectively. The physical considerations, [7], underlying (2), 
reveal that it is possible to estimate not only the average loss per cycle, but also the instantaneous loss at any given point of the hysteresis loop. In [9], it has been shown that the instantaneous loss $P_{t}(t)$ at time $t$, in a lamination of thickness $2 d$ and conductivity $\sigma$, under the condition of negligible skin effects, may be written as

$$
P_{t}(t) \equiv P_{h}(t)+P_{c}(t)+P_{e}(t) \equiv \frac{d B_{a}}{d t}\left(H_{h}+H_{c}+H_{e}\right) \equiv \frac{d B_{a}}{d t} H_{s}
$$

with

$$
H_{c}=\frac{1}{12} \sigma(2 d)^{2} \frac{d B_{a}}{d t}, H_{e}=\sqrt{\sigma G S V_{0}}\left|\frac{d B_{a}}{d t}\right|^{0.5} \operatorname{sign}\left(\frac{d B_{a}}{d t}\right),
$$

where the hysteresis field $H_{h}$ of course depend on the instantaneous induction $B_{a}$ and where moreover, for the sake of accuracy, also the internal field $V_{0}$ is allowed to vary with $B_{a}$. On the basis of (10) and (11), two simplified models may be constructed.

\subsection{Method 1: Correction of the Dynamic Field $\boldsymbol{H}_{d}$}

The first simplified model, starts from the $B_{a} H_{h}$-relation, obtained from the CPM, defined by (3) and (5), describing the quasi-static (hysteresis) behaviour of the material. To obtain the $B_{a} H_{s}$-loops, the hysteresis field $H_{h}$ is corrected by the dynamic field $H_{d}=H_{c}+H_{e}$ at each time point $t$, in order to obtain the magnetic field at the surface of the lamination, viz. $H_{s}=H_{h}+H_{d}$. Due to the dependency of $V_{0}$ on the induction $B_{m}$, the dynamic field $H_{d}$ depends on $B_{a}$ and $d B_{a} / d t$, but not on the history of the magnetic field. Therefore, for this method, it is sufficient to reconstruct the function $H_{d}(t) \equiv H_{d}\left(B_{a}, d B_{a} / d t\right)$. For the purpose of identification of the function $H_{d}$ only limit $B_{a} H_{s}$-cycles, obtained by the LM-DPM at different frequencies are compared with the quasi-static limit cycle, similar as in Figure 2, but now with $B_{m}$ equal to the saturation induction $1.9 \mathrm{~T}$.

\subsection{Method 2: Correction of the Classical Field $\boldsymbol{H}_{c}$}

The second simplified model, starts from the $B_{a}\left(H_{h}+H_{c}\right)$-relation, which is obtained from the DPM, defined by (4) and (5), and which 
describes the hysteresis and the excess losses. Here the $H_{e f}(t)$ waveform, i.e., $H_{e f}(t)=H_{h}(t)+H_{e}(t)+k_{m} B_{a}(t)$, corresponding with a sinusoidal variation of $B_{a}(t)$ with the desired amplitude $B_{m}$ and frequency $f$, must be obtained iteratively. To obtain the $B_{a} H_{s}$-loop including the three types of losses, the magnetic field $H_{h}+H_{e}$ in the previous loop is corrected by the classical field $H_{c}$ according to (11).

\section{EXPERIMENTAL VALIDATION OF THE MODELS}

In this section we discuss the validity of the simplified methods 1 and 2 as compared with the much involved combined lamination-dynamic Preisach model (LM-DPM) of Section 4 and with experimental results. As methods 1 and 2 are based upon formula (2), which presumes sinusoidal $B_{a}$-excitation, and as moreover also the function $H_{d}(t)$ is constructed under this type of excitation, we first investigate the performance of the methods 1 and 2 under sinusoidal $B_{a}$-excitation. For practical applications, however, it is also relevant to find out whether or not these methods remain reliable under non sinusoidal excitation.

In this experimental validation we considered a classical $3.2 \%$ laminated $\mathrm{SiFe}$ alloy of thickness $0.35 \mathrm{~mm}$ for which the material parameters $P(\alpha, \beta), k_{m}$ and $k_{d}$ are identified as in [10].

\subsection{Sinusoidal Excitation Conditions}

We consider 3 cases: $B_{m}=0.5$ Tesla at $\mathrm{f}=100 \mathrm{~Hz}$ (case1); $B_{m}=1$ Tesla at $\mathrm{f}=400 \mathrm{~Hz}$ (case2) and $1000 \mathrm{~Hz}$ (case3). For all cases the calculation of the variation of the induction $B$ throughout the thickness of the lamination was performed with the LM-DPM.

Figures 3-5 show the time variation of the local induction $B$ at the surface $(x=d)$ and in the middle $(x=0)$ of the lamination for the 3 cases, during one period. As expected, the influence of the skin effects on the space variation of $B$ increases with the frequency of the excitation applied. Notice that for case 1 the skin effects may be neglected, while they are significant in cases 2 and 3 . The corresponding $B_{a} H_{s}$-loops for the 3 cases are depicted in Figures 6-8. Here, the loops resulting from method 1 and 2 are compared with the loops evaluated by the LM-DPM as well as with the experimentally 


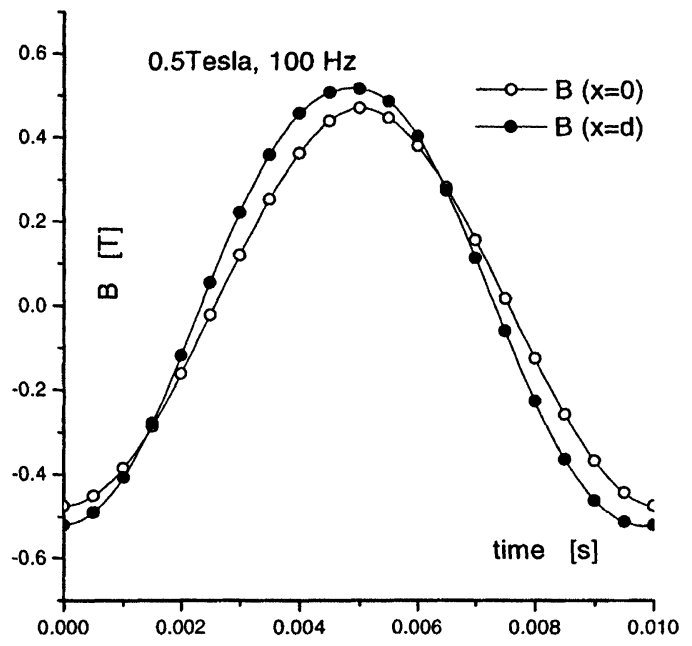

FIGURE 3 Time variation over one period of the induction $B$ at the surface and in the middle of the lamination: $B_{m}=0.5$ Tesla and $f=100 \mathrm{~Hz}$.

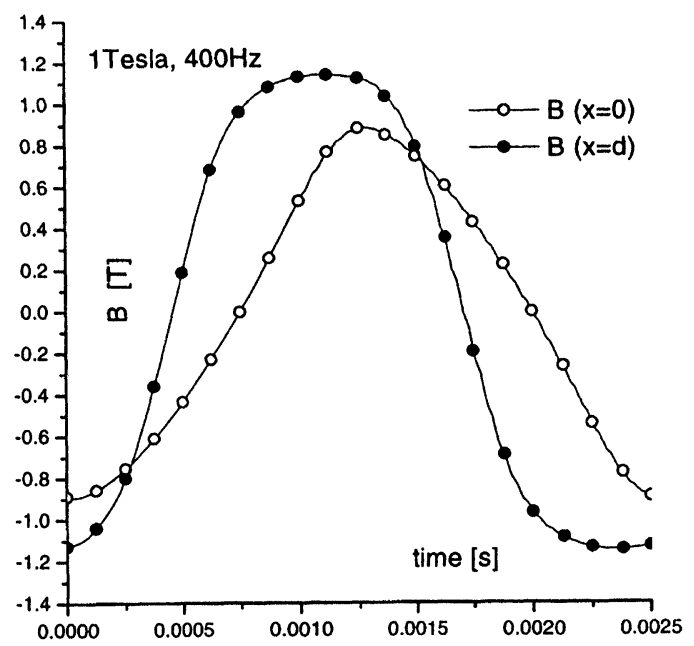

FIGURE 4 Time variation over one period of the induction $B$ at the surface and in the middle of the lamination: $B_{m}=1$ Tesla and $f=400 \mathrm{~Hz}$.

measured loops. In case 1 , where skin effects were negligible, the $B_{a} I_{5}$ loops obtained from both simplified methods coincide nearly completely with the loop obtained from the much involved LM-DPM, 


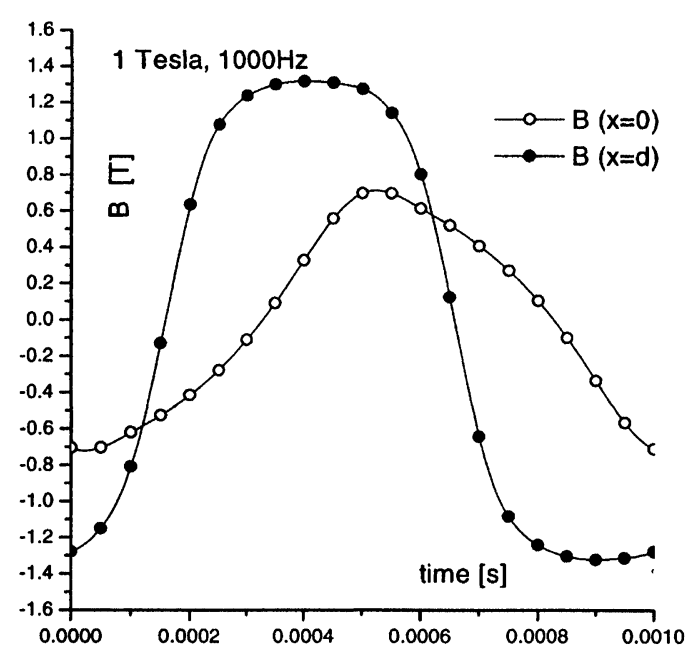

FIGURE 5 Time variation over one period of the induction $B$ at the surface and in the middle of the lamination: $B_{m}=1$ Tesla and $f=1000 \mathrm{~Hz}$.

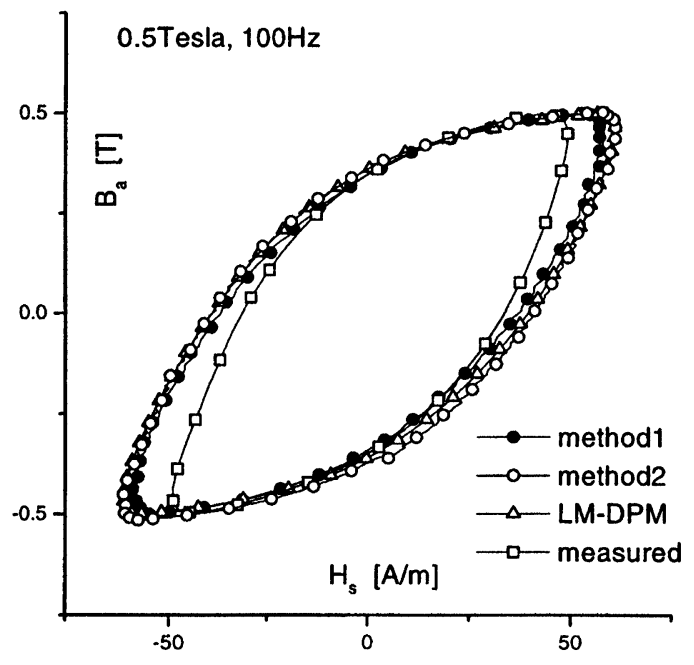

FIGURE 6 Comparison of the $B_{a} H_{s}$-loops for the methods 1 and 2 and for the LMDPM with the measured loop: $B_{m}=0.5$ Tesla, $f=100 \mathrm{~Hz}$.

itself deviating from the measured loop. This deviation is due to the limitations of the identification procedure of the material parameters in the Preisach model. Figures 7-8 reveal that in the cases 2 and 3 


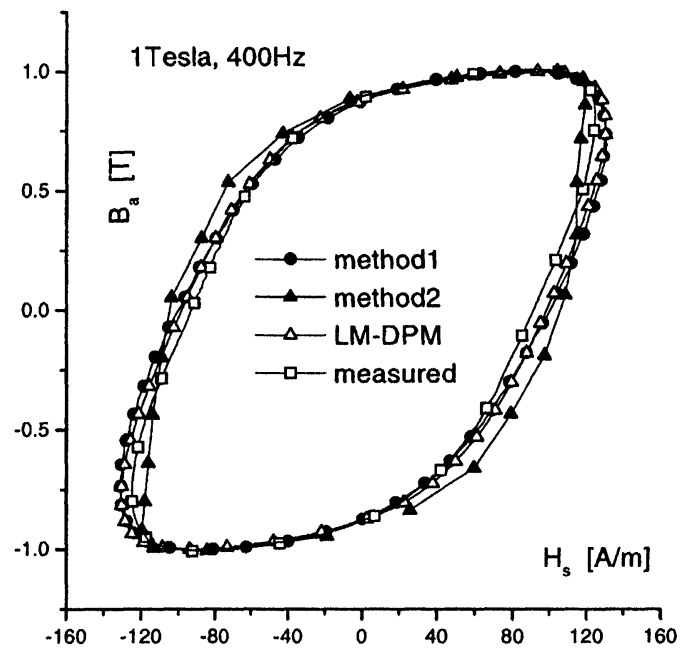

FIGURE 7 Comparison of the $B_{a} H_{s}$-loops for the methods 1 and 2 and for the LMDPM with the measured loop: $B_{m}=1$ Tesla, $f=400 \mathrm{~Hz}$.

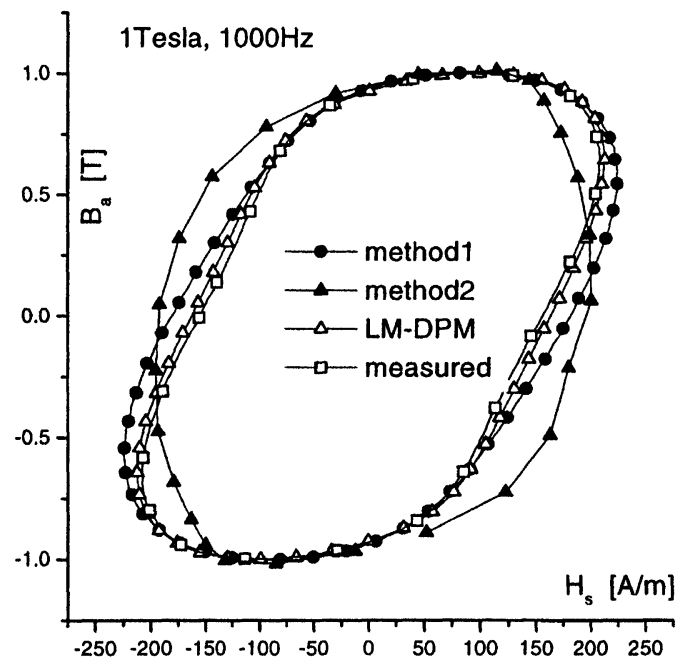

FIGURE 8 Comparison of the $B_{a} H_{s}$-loops for the methods 1 and 2 and for the LMDPM with the measured loop: $B_{m}=1$ Tesla, $f=1000 \mathrm{~Hz}$.

(where skin effects become important), the $B_{a} H_{s}$-loop obtained by method 2 deviates more from the experimental $B_{a} H_{s}$-loop than the one obtained by method 1 . Moreover, it is observed that the $B_{a} H_{s}$-loop 
obtained by method 1 is less affected by the skin effects than the loop resulting from method 2. In fact, for a moderate frequency (case 2) method 1 still leads to a $B_{a} H_{s}$-loop which is as accurate as the one from the LM-DPM, while for high skin effects (case 3) the difference between the results from method 1 and from the LM-DPM are no longer negligible. A comparison of the $B_{a} H_{s}$-loops depicted in Figures 6-8 indicates that method 2 can only be used in the low frequency case, where skin effects are negligible, while method 1 leads to fairly accurate results also in the case where skin effects appear. The latter may be understood from the fact that the identification of the function $H_{d}(t)=H_{d}\left(B_{a}, B_{a} / d t\right)$, introduced in Section 5.1, leans upon the limit $B_{a} H_{s}$-cycle at different frequencies, already taking into account the skin effects. The fact that the function $H_{d}$ which is identified by using only the limit $B_{a} H_{s}$-loops $\left(B_{m}=1.9\right.$ Tesla), gives good results for the $B_{a} H_{s}$-loops at different excitation levels (see Figs. 6-8), supports the assumption that the function $H_{d}(t)$ only depends on the instantaneous induction $B_{a}(t)$ and its derivative and not on its past values. This is not the case for $H_{h}(t)$. These considerations only concern the shape of $B_{a} H_{s}$-loops as such. However, notice that the area enclosed by the

TABLE I Comparison of the area enclosed by the $B_{a} H_{s}$-loops derived from method 1, method 2, LM-DPM and measurements

\begin{tabular}{lcccc}
\hline & LM-DPM & Method 1 & Method 2 & Measurement \\
\hline Case 1 & 62.56 & 61.32 & 64.02 & 50.76 \\
Case 2 & 348.97 & 351.62 & 354.1 & 327.31 \\
Case 3 & 568.21 & 598.14 & 602.26 & 557.98 \\
3rd, 5th & 108.67 & 110.32 & - & 100.7 \\
17th, 19th & 194.72 & 195.84 & - & 190.89 \\
\hline
\end{tabular}

TABLE II Comparison of the CPU-time per period in seconds used for the LM-DPM and the 2 simplified methods. The difference between the method 1 and method 2 is due to the iterative procedure in method 2 and the discretisation required in the Preisach plane for the dynamic Preisach model

\begin{tabular}{lccc}
\hline & LM-DPM & Method 1 & Method 2 \\
\hline Case 1 & 83 & 0.039 & 0.59 \\
Case 2 & 86 & 0.039 & 0.63 \\
Case 3 & 79 & 0.040 & 0.61 \\
3rd, 5th & 85 & 0.042 & - \\
17th, 19th & 93 & 0.041 & - \\
\hline
\end{tabular}


$B_{a} H_{s}$-loops, measuring the total electromagnetic losses, is nearly the same for method 1, method 2 and the LM-DPM, $c f$. Table I.

As shown in Table II, the CPU-time for the simplified methods is merely $1 / 2000$ of the CPU-time needed in the very accurate but much involved LM-DPM.

\subsection{Non-sinusoidal Flux Excitations}

As the higher harmonics have practical relevance towards the analysis of electromagnetic devices, we also consider non sinusoidal $B_{a^{-}}$ excitations for the assessment of the simplified method 1 . We restrict ourselves to this method as in Section 6.1 it has been found to give more accurate $B_{a} H_{s}$-loops than method 2 .

We consider two cases, viz. the case of $50 \mathrm{~Hz}$-excitation with $3 \mathrm{rd}$ and 5 th harmonics superponed, and the case of $50 \mathrm{~Hz}$-excitation with 17th and 19th harmonics superponed. From [11] we recall that these harmonics may occur in rotating electrical machines due to saturation effects (mainly harmonics 3 and 5) and due to slot effects (harmonics 17 and 19, for a given geometry). For these cases the calculation of the variation of the induction throughout the lamination thickness has been performed with the LM-DPM. In Figure 9 the results are

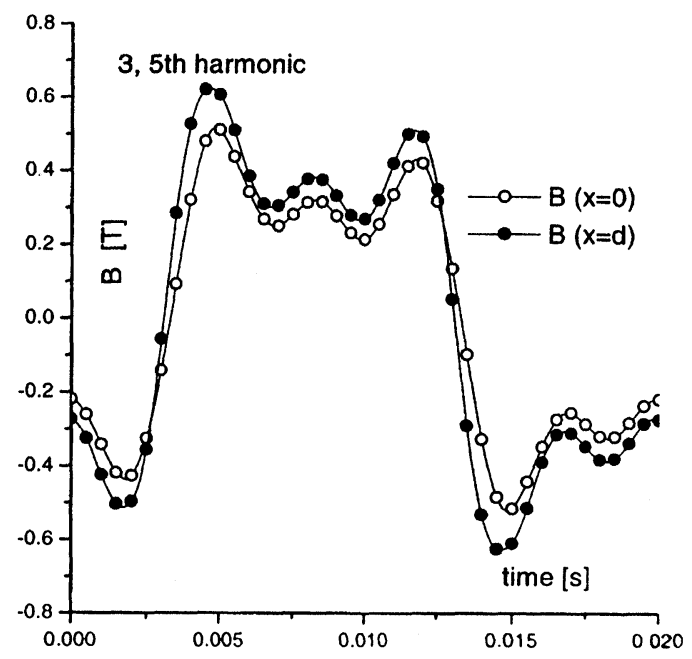

FIGURE 9 Time variation of the induction over one period at the surface and in the middle of the lamination, for the $3 \mathrm{rd}$ and the 5 th harmonics. 
depicted for the case of the 3rd and 5th harmonics (the results for the case of 17th and 19th harmonics are very similar). Skin effects have led to a space variation of the induction. In Figure 10 we compare the

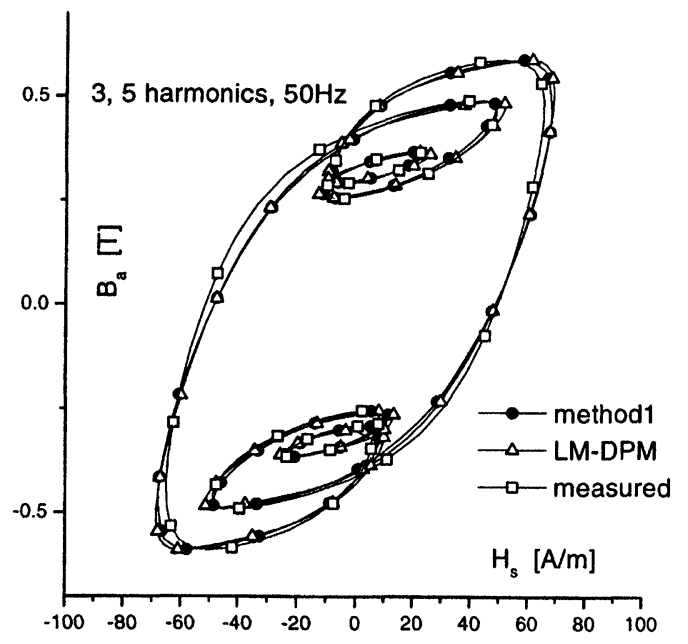

FIGURE 10 Comparison of the $B_{a} H_{s}$-loops for the method 1 and the LM-DPM with the measured loop, for the case with 3 rd and 5th harmonics.

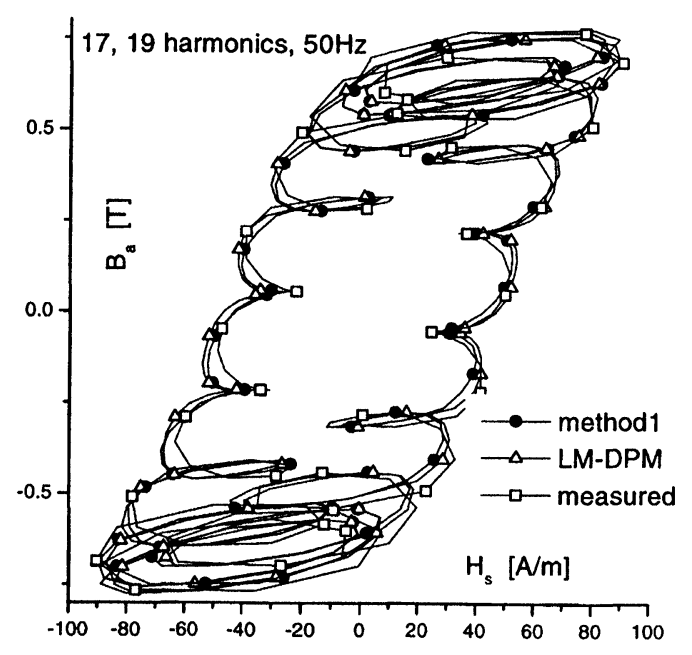

FIGURE 11 Comparison of the $B_{a} H_{s}$-loops for the method 1 and the LM-DPM with the measured loop, for the case with 17 th and 19th harmonics. 
$B_{a} H_{s}$-loops obtained by the simplified method 1, by the LM-DPM and by experimental measurements for the case of the 3rd and 5th harmonics. Figure 11 gives a similar comparison for the case of the 17th and 19th harmonics. The $B_{a} H_{s}$-loops obtained by method 1 are in good agreement with the $B_{a} H_{s}$-loops resulting from the LM-DPM, itself almost coinciding with the experimental loops. This indicates that the simplified method 1 still gives very accurate $B_{a} H_{s}$-loops and hence enclosed area (electromagnetic losses) for the case of non sinusoidal $B_{a}$-excitation.

\section{CONCLUSIONS}

In this paper 2 simplified methods were proposed to evaluate the electromagnetic behaviour of laminated SiFe-alloys by means of the statistical loss theory and the Preisach model. The $B_{a} H_{s}$-loops resulting from the 2 methods were compared with measured $B_{a} H_{s}$-loops and with the loops obtained by the numerically much involved, but accurate combined lamination model-dynamic Preisach model, as described in [5]. This LM-DPM involves 3 discretisation techniques, i.e., a time discretisation (by finite differences) and a space discretisation (by finite elements) of the problem (6)-(8) and, finally, a discretisation of the material model in the Preisach plane. The simplified methods, presented, however, are only based upon the Preisach material model itself, viz. the classical Preisach model (for method 1) and the dynamic Preisach model (for method 2). In these simplified methods the solving of the PDE is replaced by a correction technique of the magnetisation loops obtained by the respective Preisach theory, using the dynamic field $H_{d}(t)$ (method 1) and the classical field $H_{c}(t)(\operatorname{method} 2)$. This correction technique has been derived from the well established statistical magnetic loss theory, [7]. Both for the case of non-sinusoidal $B_{a}(t)$-excitation as well as for the case of sinusoidal excitation, the method 1 is found to give accurate $B_{a} H_{s}$-loops. For method 2 there is a loss of accuracy with respect to the shape of the $B_{a} H_{s}$-loops as soon as skin effects become important. However, with respect to the area enclosed by the $B_{a} H_{s}$-loops (electromagnetic losses), the discrepancy between the method 2 and the LM-DPM is much less pronounced. Finally, it should be emphasized that the simplified method 1 needs only 
$1 / 2000$ and method 2 needs only $1 / 150$ of the CPU-time that is needed for the complex LM-DPM, which should not be surprising from the arguments above.

Further research will be directed towards the extension of the methods present to the problem of $2 \mathrm{D}$ flux patterns.

\section{Acknowledgements}

Luc Dupre is a post-doctoral researcher of the Fund of Scientific Research-Flanders (F.W.O.). The authors gratefully acknowledge financial support of the 60A-project 99-200/4, the FWO-project $3604209 \mathrm{~B}$ and the IWT-STWW-project 980357.

\section{References}

[1] Preisach, F. (1935). Uber die magnetishe nachwirkung, Zeitschr. für Phys. 94, 277-302.

[2] Halliday, D. and Resnick, R. (1981). Fundamentals of Physics, John Wiley \& Sons, New York.

[3] Bertotti, G. (1992). Dynamic generalisation of the scalar Preisach model of hysteresis, IEEE Trans. on Magn., 28, 2599-2581.

[4] Philips, D., Dupre, L. and Melkebeek, J. (1994). Magneto-Dynamic Field Computation Using a Rate-Dependent Preisach model, IEEE Trans. Magn., 30, 4377-4379.

[5] Dupre, L., Van Keer, R. and Melkebeek, J. (1996). On a Magnetodynamic Model for the Iron Losses in non-oriented Steel Laminations, Journ. Phys. D, Appl. Phys. 29, 855-858.

[6] Dupre, L., Bottauscio, O., Chiampi, M., Fiorillo, F., Lo Bue, M., Melkebeek, J., Repetto, M. and von Rauch, M. (1998). Dynamic Preisach modelling of ferromagnetic laminations under distorted flux excitations, IEEE Trans. Magn., 34, $1231-1233$.

[7] Bertotti, G. (1988). General properties of Power losses in soft ferromagnetic materials, IEEE Trans. on Magn., 24, 621-630.

[8] Lammeraner, J. and Stafl, M. (1996). Eddy Currents, Iliffe Books Ltd, London.

[9] Bertotti, G. (1998). Hysteresis in Magnetism, Academic Press, San Diego.

[10] Dupre, L., Van Keer, R. and Melkebeek, J. (1997). Modelling and identification of the iron losses in non-oriented steel laminations using the Preisach theory, IEE Proceedings - Electric Power Applications, 144, 227-234.

[11] Van Keer, R., Dupre, L. and Melkebeek, J. (1999). Computational Methods for the Evaluation of the Electromagnetic Losses in Electrical Machinery, Archives Comp. Meth. Engin., 5, 385-443. 


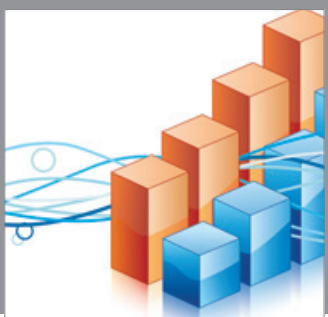

Advances in

Operations Research

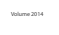

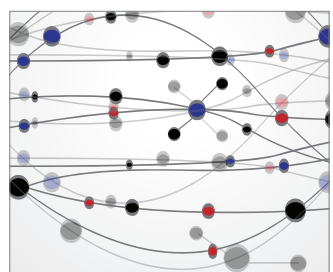

\section{The Scientific} World Journal
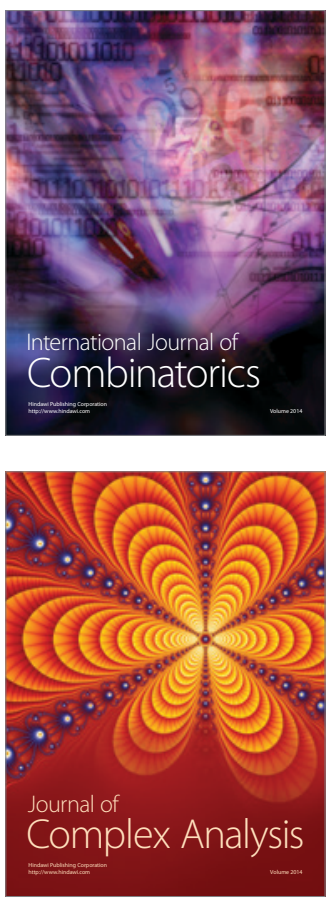

International Journal of

Mathematics and

Mathematical

Sciences
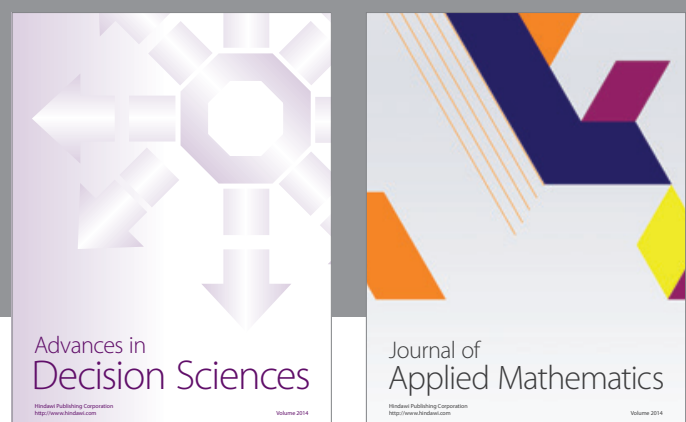

Journal of

Applied Mathematics
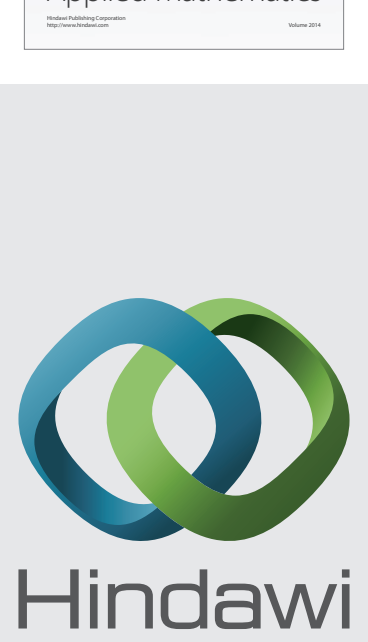

Submit your manuscripts at http://www.hindawi.com
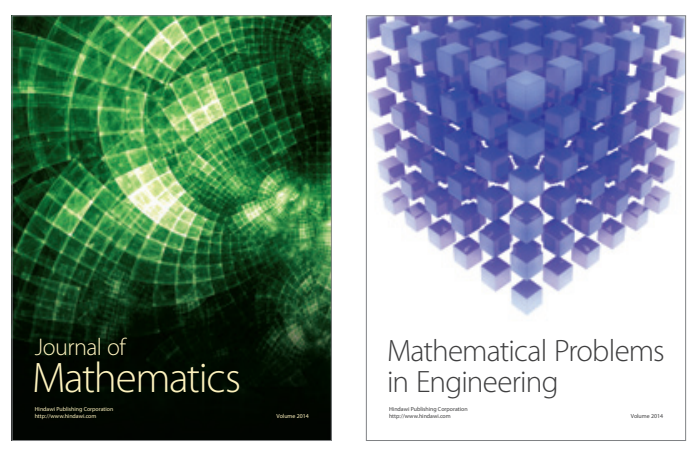

Mathematical Problems in Engineering
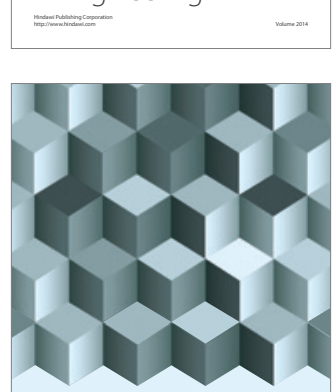

Journal of

Function Spaces
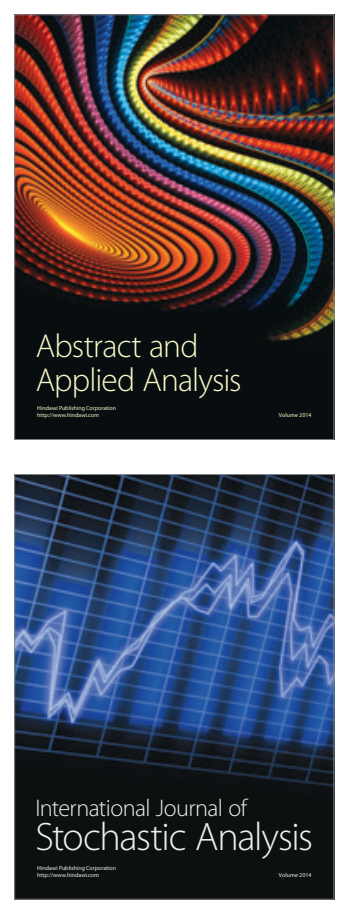

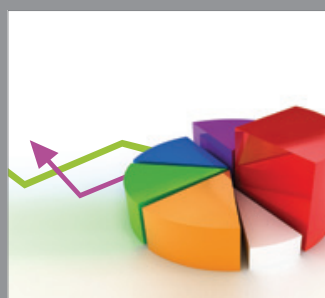

ournal of

Probability and Statistics

Promensencen
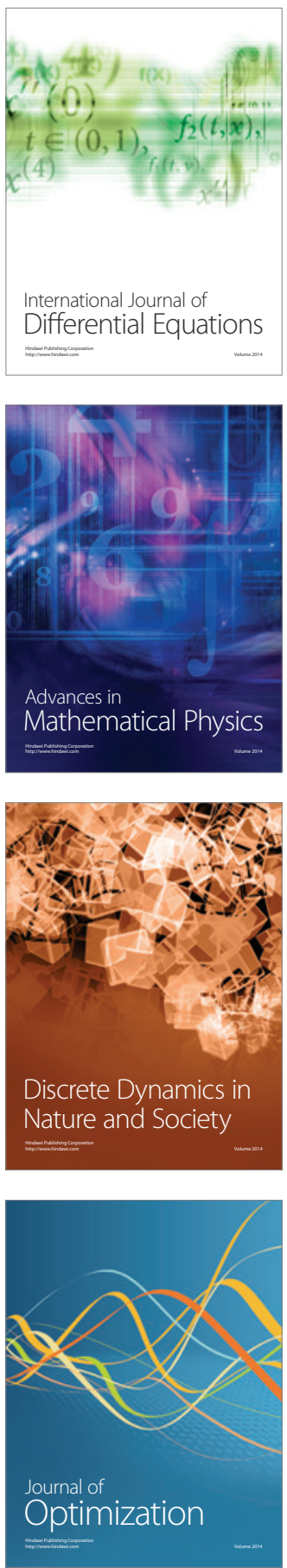